\title{
EXPERIMENTAL TOUR-BASED TRAVEL DEMAND MODELS
}

\author{
Demetrio C. Festa, Daniela Condino e Gabriella Mazzulla \\ Department of Regional Planning - University of Calabria (Italy) \\ Via P.Bucci, 87036 Rende (CS) \\ Tel. +39.984.496752-81 \\ Fax $+39.984 .496782-87$ \\ e-mail: dc.festa@unical.it -condino@unical.it -g.mazzulla@unical.it
}

\section{ABSTRACT}

In this paper some experimental sequential models for the simulation of trip-chains are presented; the models have been calibrated on the base of a survey made in a medium-sized town.

This work is part of a research, effected by the Department of Regional Planning of the University of Calabria, to forecast travel demand and to analyse travel behaviour of the transport system users. (Festa et al., 2000).

The travel choices of individuals have been reproduced simulating the decisional process in sequential steps, by models based on the random utility theory.

The tour generation models, proposed by Festa et al. 2001, have been once again calibrated introducing a new set of variables in the systematic utility function of the alternatives of choice.

Some models for the travel type choice simulation are also presented; the models have a Binomial Logit functional structure, with trip-tour and trip-chain as choice alternatives.

\section{SEQUENTIAL MODELS FOR TRAVEL DEMAND ESTIMATION}

In literature, various structures of models, finalized to estimate the travel demand, are reported. Considered the complexity of the phenomenon and the variety of the adopted formulations, it is difficult to make a rigorous classification. However, there are two main approaches: a global approach, which reproduces the phenomenon by a single model, and a sequential approach which, by a system of sub-models, replicates the decisional process in successive stages which represent the corresponding dimensions of choice.

The sequential models differ for the elementary unit which represents the travel demand. This unit, in order of complexity, can be identified with the single movement from an origin to a destination (trip), the sequence of trips based at home (tour), or the daily/weekly travel program (pattern).

The trip-based models are built on the hypothesis that the choices related to the various movements are not mutual conditioned. A similar travel demand model system was 
developed for the MTC (Metropolitan Transportation Commission), in the metropolitan planning organization for the S. Francisco area (Ben-Akiva M., Lerman S.R., 1985). An analogous formulation has been proposed in the Transport Project of the Italian National Research Council, to forecast the travel demand in medium-sized Italian towns (Cascetta, 1990).

The tour-based models, unlike the previous models, take account of the time and space constraints among the trips of the same tour. The tours are generally characterized through the Primary Destination, defined as the destination in which the most important activity is made. Recently, various behavioural travel demand model systems based on the random utility theory have been developed; these models differ each others for the definition of the dimensions of choice. The better-structured models simulate, in various stages, the tour generation and frequency, the space distribution (primary and secondary destination), the tour type and the mode choice (Cascetta, 1998). Other formulations represent more dimensions of choice through a single aggregate submodel, like the destination and mode choice (Bowman and Ben-Akiva, 2000).

The daily or weekly travel-pattern demand models take account of the interactions among the tours made during the reference period; therefore, they have a more complex formulation. Some authors propose regressive models, in which the travel program depends on the activity program; the explicative variables, both of the travel behaviour and activities participation, are assumed as endogenous variables, and are expressed in function of the individual and household socio-economic characteristics (Lu and Pas, 1999; Golob, 2000).

\section{A MODELS SYSTEM FOR THE SIMULATION OF THE TRAVEL CHOICES}

\subsection{General structure}

The calibration and validation results of behavioural random utility models, for the trip generation and the tour type choice, are presented. The introduced formulations are placed in a system of models that simulates the decisional process in successive stages, according to the structure proposed in Cascetta (1998). In this context, between the generation and the tour type choice, the model of primary destination choice is placed; this model has not been analysed in this stage of research.

The proposed models have been calibrated on the base of a survey, made in a medium-sized urban area (the town of Cosenza in the Southern Italy), on a wide sample of families (Festa et alii, 2000).

The tours have been characterized by the destination in which the major activity has been done. The primary destination has been chosen attributing to the activities a different hierarchical level, in function of the constraint degree which they determine in the space and the time. In particular, four hierarchical levels of activity have been specified, attributing to work in a permanent address and study activities, more time-space constrained, the higher degree; the work activities, made in a non habitual workplace, have been sited to the 
immediately inferior level; non work and non study activities have been unified in two different hierarchical levels, classifying the personal affairs and the person taking activities more constrained than the recreation and shopping ones.

Finally, the calibrated models estimate the probability to generate a tour for the predominant activity and, subsequently, the probability to make some other activities during the same tour.

\subsection{Tour generation models}

In this paper, models have been specified and calibrated for the generation of tours based at home, as tours oriented to shopping, to recreation activity, to take some person, to personal affairs. No generation models for work and study activities are presented.

The specified alternatives have a Binomial Logit structure with two alternatives of choice: to make a travel (TRIP) and not to make it (NOTRIP).

The systematic utility of the alternatives depends both on individual socio-economic variables, as age (AGE), sex (SEX), employment (EMP) and professional level (PLEV), and on structure and socio-economic condition of the family. The household structure has been described through two variables: the first one represents the role played in the household and attributes a greater weight to the role of head of the family or consort (HEADFAM); the second variable has been obtained by the ratio between the number of children (members with age under 14) and the total number of household members (CHILD). The socioeconomic conditions of the family have been represented through a variable valued by the ratio between the number of owned cars and the number of licensed members (CAR).

Two variables have been specified to take account both of the temporal constraints, which limit the individual choice in the generative phase of the travel, and the interaction among the individual and the other family members. The first variable is represented through the number of other home-based trips made by the single person (OP); the second one has been obtained as the number of trips, also Non home-based, realized by other household members for the analysed purpose (OO) (Damm, 1982; Biggiero, 1991).

Finally, the specified functional structure is the following:

$$
\begin{aligned}
V_{\text {TRIP }}= & \beta_{\text {SEX }} \cdot S E X+\beta_{A G E} \cdot A G E+\beta_{\text {EMP }} \cdot E M P+\beta_{\text {PLEV }} \cdot P L E V+\beta_{\text {HEADFAM }} \cdot \text { HEADFAM }+ \\
& \beta_{\text {CHILD }} \cdot C H I L D+\beta_{\text {CAR }} \cdot C A R \\
V_{\text {NOTRIP }}= & \beta_{\text {OP }} \cdot \text { OP }+\beta_{O O} \cdot O O+\beta_{\text {NoTRIP }} \cdot \text { NOTRIP }
\end{aligned}
$$

The sample, used for the calibration of the models, is constituted by 2,005 persons, of which 1,438 have realized some trip in the survey period. In the typical weekday, the interviewed persons have realized in total 6,172 trips, organized in 2,521 tours. Of these, 1,081 are oriented to work or study, 312 are realized for shopping, 420 for recreation activity, 231 to take a person, 346 for personal affairs and 131 for other purposes. 
For each purpose, two models have been calibrated: a complete model, that considers all variables present in the functional structure and the best model, that considers only the variables which satisfy the t-Student test. All the calibrated models verify the statistical tests on the goodness of fit; in particular the $\bar{\rho}^{2}$ statistics assume values between 0.3745 and 0.6061 , obtained for the "taking persons" purpose. For example, the calibration results of the generation model of trips oriented to shopping are presented in table 1.

Table 1. Models for the generation of tours oriented to shopping

\begin{tabular}{|c|c|c|c|c|c|c|c|c|c|}
\hline \multirow{3}{*}{ 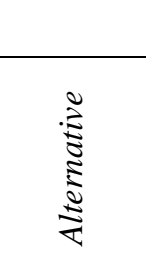 } & \multirow{3}{*}{$\frac{\sqrt{5}}{\frac{5}{0}}$} & \multicolumn{3}{|c|}{ Model with all variables } & \multicolumn{5}{|c|}{ Model with significant variables } \\
\hline & & \multirow{2}{*}{\multicolumn{2}{|c|}{ 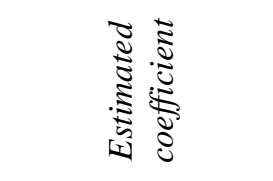 }} & \multirow{3}{*}{ 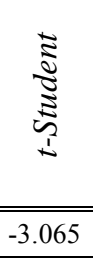 } & \multirow{2}{*}{\multicolumn{2}{|c|}{ 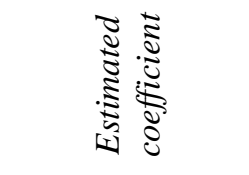 }} & \multirow{2}{*}{ 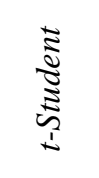 } & \multicolumn{2}{|c|}{$\%$ Choice } \\
\hline & & & & & & & & Observed & Modelled \\
\hline \multirow{7}{*}{ TRIP } & SEX & $\beta_{\mathrm{SEX}}$ & -0.4531 & & $\beta_{\mathrm{SEX}}$ & -0.4906 & -3.381 & \multirow{7}{*}{$14.91 \%$} & \multirow{7}{*}{$14.92 \%$} \\
\hline & AGE & $\beta_{\mathrm{AGE}}$ & 0.1780 & 1.076 & $\beta_{\mathrm{AGE}}$ & - & - & & \\
\hline & EMP & $\beta_{\mathrm{EMP}}$ & -0.6597 & -3.445 & $\beta_{\mathrm{EMP}}$ & -0.6522 & -3.885 & & \\
\hline & PLEV & $\beta_{\mathrm{PLEV}}$ & -0.2816 & -0.986 & $\beta_{\mathrm{PLEV}}$ & - & - & & \\
\hline & HEADFAM & $\beta_{\text {HEADFAM }}$ & 1.5410 & 8.309 & $\beta_{\text {HEADFAM }}$ & 1.6110 & 9.310 & & \\
\hline & CHILD & $\beta_{\text {CHILD }}$ & -1.4090 & -3.299 & $\beta_{\text {CHILD }}$ & -1.3770 & -3.247 & & \\
\hline & CAR & $\beta_{\mathrm{CAR}}$ & 0.4219 & 2.429 & $\beta_{\mathrm{CAR}}$ & 0.4251 & 2.463 & & \\
\hline \multirow{3}{*}{ NOTRIP } & OP & $\beta_{\mathrm{OP}}$ & 0.2184 & 2.668 & $\beta_{\mathrm{OP}}$ & 0.2167 & 2.649 & \multirow{3}{*}{$85.09 \%$} & \multirow{3}{*}{$85.08 \%$} \\
\hline & $\mathrm{OO}$ & $\beta_{\mathrm{Oo}}$ & -0.4363 & -7.165 & $\beta_{\mathrm{OO}}$ & -0.4407 & -7.242 & & \\
\hline & NoTRIP & $\beta_{\text {NoTRIP }}$ & 2.5440 & 13.67 & $\beta_{\text {NoTRIP }}$ & 2.5380 & 13.65 & & \\
\hline \multicolumn{2}{|c|}{$\log L(\boldsymbol{\beta})$} & \multicolumn{3}{|c|}{-746.02} & \multicolumn{5}{|c|}{-747.12} \\
\hline \multicolumn{2}{|c|}{$\operatorname{LogL}(\mathbf{0})$} & \multicolumn{3}{|c|}{$-1,389.76$} & \multicolumn{5}{|c|}{$-1,389.76$} \\
\hline \multicolumn{2}{|c|}{ LR } & \multicolumn{3}{|c|}{$1,287.48\left(\chi^{2}\right.$ with 10 d.o.f.: 18.31$)$} & \multicolumn{5}{|c|}{$1,285.28\left(\chi^{2}\right.$ with 8 d.o.f.: 15.51$)$} \\
\hline \multicolumn{2}{|c|}{$\bar{\rho}^{2}$} & \multicolumn{3}{|c|}{0.4560} & \multicolumn{5}{|c|}{0.4567} \\
\hline \multicolumn{2}{|c|}{$\%$ RIGHT } & \multicolumn{3}{|c|}{$85.34 \%$} & \multicolumn{5}{|c|}{$85.24 \%$} \\
\hline
\end{tabular}

In the sample, there are 299 persons that have made tours for this purpose and, in particular, 13 of these have made two tours for the same raison. The variables AGE and PLEV have not resulted statistically significant; the signs assumed by coefficients indicate that the persons of female sex, unemployed and that play a predominant role in the household, are more motivated to make the tours for shopping; in opposite, the presence of children and the making other tours of this type, in the same day, represent a deterrent. The negative sign assumed by variable $\mathrm{OO}$ can be interpreted as an index of the aptitude to make shopping with other household members.

\subsection{Tour type choice models}

Tour type choice models, used to characterize the modalities with which the persons organize their own travel program, have been specified and calibrated. The specified models 
have a Binomial Logit structure, in which the alternatives are represented by the choice to make a tour with a single sojourn (TRIP-TOUR) or a tour with two or more sojourns (TRIPCHAIN); a sojourn can be defined as an intermediate stop in the travel that is oriented to make an activity.

The types of variables considered in the utility function are:

- individual socio-economic variables, as age (AGE), sex (SEX), employment (EMP) and professional level (PLEV);

- variables related to the household structure, as the role played in the family (HEADFAM) and the ratio between the number of children and the total number of members (CHILD);

- variables representing the temporal constraints, as the departure time from home (DEPTIME), the duration of activity done in the primary destination (ACTDUR), the number of other tours made from the person in the same day (OT), the total travel time spent to go to the primary destination (TTIME);

- an index of accessibility, obtained as difference between the active accessibility of primary destination and active accessibility of residence zone (ACC);

- variables of interaction between the activity program and the travel program, as the type of major activity (ACT) and the mode used to go to the primary destination (MODE).

The active accessibility is generally expressed as the maximum value of utility obtained from the dimension of the destination choice. In this work, no spatial distribution model has been calibrated, then, the accessibility measure has been expressed by the reciprocal value of the total generalized cost that the person must bear to go to all other destinations, starting from his own zone.

The tour type models, on the contrary of the calibrated travel generation models, are not diversified on the activity type. In this perspective, the activity variables represent the tendency to combine some activities during the same tour.

The specified functional structure is now the following:

$$
\begin{gathered}
V_{\text {TRIP }-T O U R}=\beta_{\text {SEX }} \cdot S E X+\beta_{\text {AGE }} \cdot A G E+\beta_{E M P} \cdot E M P+\beta_{\text {PLEV }} \cdot P L E V+\beta_{\text {HEADFAM }} \cdot \text { HEADFAM }+ \\
\beta_{\text {CHILD }} \cdot C H I L D+\beta_{\text {DEPTIME }} \cdot D E P T I M E+\beta_{\text {ACTDUR }} \cdot A C T D U R+\beta_{\text {OT }} \cdot \text { OT }+\beta_{A C T} \cdot A C T \\
V_{\text {TRIP-CHAIN }}=\beta_{M O D E} \cdot M O D E+\beta_{A C C} \cdot A C C+\beta_{\text {TTIME }} \cdot \text { TTIME }+\beta_{\text {CHAIN }} \cdot C H A I N
\end{gathered}
$$

The sample used for the calibration of the tour type choice model is constituted by the tours having the primary destination in Cosenza. These tours are in total 1,821, of which 1,339 are trip-tours and 482 are trip-chains. Analogously to the generation models, the results of the calibration of the complete model and the best model are presented (table 2). For the last one, LR and \%RIGHT statistical tests on the goodness of fit are fully verified; the $\bar{\rho}^{2}$ statistic is approximately equal to 0.3 . 
Table 2. Models for the tour type choice

\begin{tabular}{|c|c|c|c|c|c|c|c|c|c|}
\hline \multirow{3}{*}{ 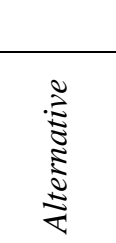 } & \multirow{3}{*}{$\begin{array}{c}\frac{1}{0} \\
\frac{0}{0} \\
0\end{array}$} & \multicolumn{3}{|c|}{ Model with all variables } & \multicolumn{5}{|c|}{ Model with significant variables } \\
\hline & & \multirow{2}{*}{\multicolumn{2}{|c|}{ 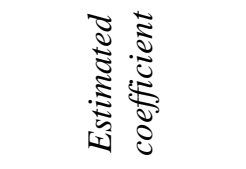 }} & \multirow{3}{*}{ 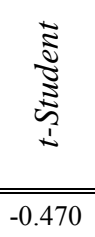 } & \multirow{2}{*}{\multicolumn{2}{|c|}{ 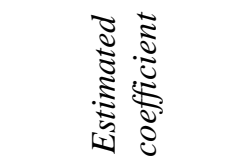 }} & \multirow{2}{*}{ 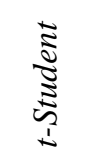 } & \multicolumn{2}{|c|}{$\%$ Choice } \\
\hline & & & & & & & & Observed & Modelled \\
\hline \multirow{10}{*}{$\begin{array}{c}T R I P \\
T O U R\end{array}$} & SEX & $\beta_{\mathrm{SEX}}$ & -0.0601 & & $\beta_{\mathrm{SEX}}$ & - & - & \multirow{10}{*}{$73.53 \%$} & \multirow{10}{*}{$73.53 \%$} \\
\hline & AGE & $\beta_{\mathrm{AGE}}$ & -0.3675 & -2.389 & $\beta_{\mathrm{AGE}}$ & -0.3556 & -2.333 & & \\
\hline & EMP & $\beta_{\mathrm{EMP}}$ & -0.4408 & -2.619 & $\beta_{\mathrm{EMP}}$ & -0.5285 & -3.578 & & \\
\hline & PLEV & $\beta_{\text {PLEV }}$ & -0.2702 & -1.382 & $\beta_{\text {PLEV }}$ & - & - & & \\
\hline & HEADFAM & $\beta_{\text {HEADFAM }}$ & 0.5932 & 3.698 & $\beta_{\text {HEADFAM }}$ & 0.5924 & 3.805 & & \\
\hline & CHILD & $\beta_{\text {CHILD }}$ & -0.8930 & -2.517 & $\beta_{\text {CHILD }}$ & -0.8886 & -2.518 & & \\
\hline & DEPTIME & $\beta_{\text {DEPTIME }}$ & 0.8223 & 4.925 & $\beta_{\text {DEPTIME }}$ & 0.8265 & 4.969 & & \\
\hline & ACTDUR & $\beta_{\text {ACTDUR }}$ & 0.5884 & 13.05 & $\beta_{\text {ACTDUR }}$ & 0.5889 & 14.25 & & \\
\hline & OT & $\beta_{\text {от }}$ & 0.2540 & 3.129 & $\beta_{\text {От }}$ & 0.2535 & 3.152 & & \\
\hline & $\mathrm{ACT}$ & $\beta_{\mathrm{ACT}}$ & -0.0089 & -0.058 & $\beta_{\mathrm{ACT}}$ & - & - & & \\
\hline \multirow{4}{*}{$\begin{array}{c}\text { TRIP } \\
\text { CHAIN }\end{array}$} & MODE & $\beta_{\mathrm{MODE}}$ & 0.2620 & 1.967 & $\beta_{\text {MODE }}$ & 0.2689 & 2.036 & \multirow{4}{*}{$26.47 \%$} & \multirow{4}{*}{$26.47 \%$} \\
\hline & $\mathrm{ACC}$ & $\beta_{\mathrm{ACC}}$ & 0.7580 & 3.511 & $\beta_{\mathrm{ACC}}$ & 0.7547 & 3.525 & & \\
\hline & TTIME & $\beta_{\text {TTIME }}$ & 0.5103 & 5.795 & $\beta_{\text {TTIME }}$ & 0.5095 & 5.810 & & \\
\hline & CHAIN & $\beta_{\text {CHAIN }}$ & 1.9010 & 3.336 & $\beta_{\text {CHAIN }}$ & 1.9470 & 3.509 & & \\
\hline \multicolumn{2}{|c|}{$\log L(\boldsymbol{\beta})$} & \multicolumn{3}{|c|}{-876.17} & \multicolumn{5}{|c|}{-877.27} \\
\hline \multicolumn{2}{|c|}{$\operatorname{LogL}(\mathbf{0})$} & \multicolumn{3}{|c|}{$-1,262.22$} & \multicolumn{5}{|c|}{$-1,262.22$} \\
\hline \multicolumn{2}{|c|}{ LR } & \multicolumn{3}{|c|}{772.10 ( $\chi^{2}$ with 14 d.o.f.: 23.69$)$} & \multicolumn{5}{|c|}{$769.90\left(\chi^{2}\right.$ with 11 d.o.f.: 19.68$)$} \\
\hline \multicolumn{2}{|c|}{$\bar{\rho}^{2}$} & \multicolumn{3}{|c|}{0.2948} & \multicolumn{5}{|c|}{0.2963} \\
\hline \multicolumn{2}{|c|}{ \%RIGHT } & \multicolumn{3}{|c|}{$78.09 \%$} & \multicolumn{5}{|c|}{$78.20 \%$} \\
\hline
\end{tabular}

The variables SEX and AGE have not resulted statistically significant, like the variable ACT. This last result can be indicative of how much the type of predominant activity is not influential on the choice to realize a trip-tour or a trip-chain. The more significant variables are ACTDUR, TTIME and DEPTIME, that is the variables inserted in the model in order to represent temporal constraints.

The signs assumed by coefficients indicate that the choice to realize tour-chains is preferred by persons aged between 36 and 60, employed, with children and that use, in the travel, an individual transport mode. The probability to make a tour-chain increases, both with the rise of the accessibility of the primary destination from the residence zone, and with the rise of the total travel time.

\section{REFERENCES}

Ben-Akiva M., Lerman S.R. (1985), Discrete Choice Analysis: Theory and Application Travel Demand, MIT Press, Cambridge, Massachusetts.

Biggiero L. (1991), Un modello comportamentale per la generazione degli spostamenti non sistematici in area urbana, Trasporti e Trazione n.4. 
Bowman J.L., Ben-Akiva M. (2000), Activity-based disaggregate travel demand model system with activity schedules, Transportation Research Vol. 35A.

Cascetta E. (1990), Metodi quantitativi per la pianificazione dei sistemi di trasporto, CEDAM, Padova

Cascetta E. (1998), Teoria e metodi dell'ingegneria dei Sistemi di Trasporto, UTET, Torino.

Cascetta E., Biggiero L. (1997), Integrated models for simulating the Italian passenger transport system, Eighth Symposium on Transportation Systems, Chania, Greece.

Damm D. (1982), Parameters of activity behavior for use in travel analysis, Transportation Research Vol. 16A.

Festa D.C., Mazzulla G. e Condino D. (2000), Analisi sperimentale delle catene di spostamenti in una città di medie dimensioni, IX convegno SIDT, Reggio Calabria.

Festa D.C., Mazzulla G. e Condino D. (2001), Nuova città, nuova mobilità, Seconda conferenza nazionale su "Informatica e pianificazione urbana e territoriale" - INPUT2001, Isole Tremiti (FG).

Golob T. F. (2000), A simultaneous model of household activity participation and trip chain generation, Transportation Research Vol. 34B.

Lu X., Pas E. I. (1999), Socio-demographics, activity participation and travel behavior, Transportation Research Vol. 33A. 